\title{
CARPHOLOGY by Rajendra
}

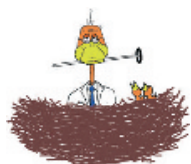

Pract Neurol 2007; 7: 276

\section{Aspirin and cognition}

Long-term use of low dose aspirin does not protect against cognitive decline in healthy and mostly white women aged 65 or more. This is the finding of a randomised, doubleblind, placebo-controlled trial in which low dose aspirin was given to a subset of 6377 women participating in the Women's health study. At the initial assessment (mean 5.6 years after randomisation) cognitive performance in the aspirin group was similar to that of the placebo group (mean difference in global score -0.01, 95\% Cl -0.04 to 0.02). According to the authors this trial does not support previous laboratory and epidemiological evidence that suggested that aspirin and other antiinflammatory drugs may be protective against dementia.

BMJ 2007;334:987.

\section{Herpes simplex encephalitis}

Herpes simplex encephalitis continues to be associated with poor long-term outcomes despite treatment with intravenous aciclovir. This is the finding of a simple prospective register-based observational study of children done in Toronto, Canada. Of 322 children with acute encephalitis, 16 had herpes simplex encephalitis. Herpes simplex virus polymerase chain reaction studies done on cerebrospinal fluid were negative initially in two children but became positive when the test was repeated later. All patients were treated with intravenous acyclovir. Neurological sequelae occurred in 11 children, of whom seven had seizures and four had developmental delays. None of the children died.

Pediatrics 2007;119:e399-407

\section{Giant cell arteritis}

Patients with giant cell arteritis are dependent on glucocorticosteroids for their treatment and any new treatment that reduces this dependence is welcome. Unfortunately, infliximab, a monoclonal antibody against tumour necrosis factor, was found to be ineffective in a small placebo-controlled trial when it was given alone or along with steroids. The 44 patients in the study had newly diagnosed giant cell arteritis and were in a glucocorticosteroid-induced remission. The relapse rates did not differ significantly and the placebo-controlled trial was stopped early. The authors say that although the trial is too small to draw definitive conclusions, it provides evidence that using infliximab in these patients is unlikely to be of great benefit.

Ann Intern Med 2007;146:621-30.

\section{Malignant glioma}

The prognosis for patients with malignant gliomas is poor so any intervention that improves survival without affecting quality of life or cognition is welcome.

Radiotherapy resulted in a modest improvement in survival in patients aged 70 years and over with newly diagnosed glioblastoma, in a multicentre randomised controlled trial done on over 80 patients. At a median follow-up of 21 weeks, the median survival for the patients who received radiotherapy plus supportive care was 29.1 weeks, as compared with 16.9 weeks for the patients who received supportive care alone. The intervention did not reduce their quality of life or cognition. The trial was stopped early because of the favourable results.

N Engl J Med 2007;356:1575-6.

\section{Preventing venous thromboembolism}

Enoxaparin is preferable to unfractionated heparin to prevent venous thromboembolism in patients with acute ischaemic stroke. This is the finding of an open-label randomised study involving over 1700 patients, which was sponsored by SanofiAventis. The study used a composite endpoint consisting of symptomatic or asymptomatic deep venous thrombosis, symptomatic pulmonary embolism, or fatal pulmonary embolism. Primary safety endpoints were symptomatic intracranial haemorrhage, major extracranial haemorrhage, and all-cause mortality. Enoxaparin reduced the risk of venous thromboem lism by $43 \%$ compared with unfraction heparin while the occurrence of any bleeding was similar in the two group Lancet 2007;369:1347-55.

\section{New poliomyelitis virus}

The newer high potency monovalent o type 1 poliovirus vaccine ( $\mathrm{mOPV} 1)$ is three times more effective than the conventi\&fal trivalent oral polio vaccine, according $\underset{N}{\infty} \mathrm{a}$ study done under field conditions in India. Poliomyelitis persists in some parts of the world including parts of India and eradicating the illness has proved challengi Researchers tested the protective efficasy of mOPV 1 and of the conventional vaccithe in a case-control study involving over 280 matched case-control pairs of confirm cases of poliomyelitis caused by type 1 i poliovirus and cases of non-polio acut flaccid paralysis in India. The authors that achieving high coverage with this vaccine in areas of persistent polioviru를 transmission should substantially impreve the probability of rapidly eliminating transmission of the disease.

Lancet 2007;369:1356-62.

\section{Eradicating or controlling polio?}

An economic analysis in the same issu the Lancet argues that although eradic tion of poliomyelitis is very expensive the much preferred option to a seemi low-cost policy aimed at controlling poliomyelitis that goes on for several y The authors base their arguments on dynamic model based on endemic area India. The analysts say that the world $\mathbb{D}$ should complete eradication now. The other option, of controlling the numberof cases, will cost more in terms of money number of cases. And there is also the that we may not have another chance $\overrightarrow{\text { to }}$ eradicate poliomyelitis.

Lancet 2007;369:1363-71. 